\title{
Revealing the gamma-ray jet in a black hole binary
}

\author{
Martin J. Hardcastle ${ }^{1}$ \\ ${ }^{1}$ School of Physics, Astronomy and Mathematics, University of Hertfordshire, College Lane, \\ Hatfield AL10 9AB, UK. E-mail: m.j.hardcastle@herts.ac.uk
}

\section{Novel techniques in gamma-ray polarimetry allow gamma-ray emission from a black-hole binary in the Milky Way to be associated with a jet.}

One of the most important advances in high-energy astrophysics over the past decade has been the detailed understanding that has emerged of the nature of binary stellar systems in which material from an ordinary star is being accreted on to a compact object, either a neutron star or a black hole[1,2]. These systems are called 'X-ray binaries' because emission from the accretion disk and associated material appears very bright in X-rays, but in recent years it has become clear that a substantial amount of the energy generated as matter is accreted can be ejected in the form of relativistic jets which inject energy into the environment around the binary system and which give the objects their alternative name of 'microquasars' [3,4]. As that name suggests, X-ray binaries may be offering us an opportunity to study analogs of the much more powerful, but much more distant, relativistic outflows associated with the supermassive black holes at the centers of active galaxies, and to watch them evolve over timescales that are compatible with a human lifetime. But direct measurements of the properties of the jets in X-ray binaries have been rather elusive to date. In this issue, Laurent et al. [5] show that gamma-ray polarization measurements can be used to make a convincing case for a jet origin for some of the gamma-ray emission observed from the prototypical X-ray binary, Cygnus X-1. 
Cygnus X-1 is a binary pair formed of a massive star ( $\sim 20$ times the mass of the Sun $)$ and a black hole approximately 10 times the mass of the sun [6], located at a distance around $2 \mathrm{kpc}$ [7]. It is one of the brightest X-ray sources in the sky, and was one of the first to be discovered [8]. Emission from a relatively faint jet is seen in high-resolution radio observations [9] but the clearest evidence that a powerful outflow is present was provided by Gallo et al. [4] who showed that a spectacular 5-pc 'bubble' has been blown in the interstellar medium by a jet which must be carrying a power 2000 times greater than the radiative luminosity of the Sun. This jet power is much more than what would have been estimated from the observations of the radio emission — indeed, there is a real problem reconciling these observations [10]. The results of [5], by providing another method of 'seeing' emission from the jet directly, should help us to understand the nature of the outflow in Cygnus X-1.

Laurent et al. have made this advance by using the IBIS instrument, on board the European gamma-ray satellite observatory INTEGRAL, to measure the polarization of the gammaray emission from Cygnus X-1 in the energy range 250-2000 keV. Although polarimetry is an essential tool for astronomers at longer wavelengths, it is very hard to make polarization measurements in the X-ray and gamma-ray. IBIS, which is primarily an imaging instrument, can be used as a gamma-ray polarimeter [11] by exploiting the fact that Compton scattering that takes place internal to the instrument is (weakly) dependent on the polarization angle of the incoming photons: by measuring the scattering angles of Compton-scattered photons, the polarization angle and degree of polarization of a source can be inferred. This is crucial to the analysis of Cygnus X-1, because different astronomical sources of high-energy photons may have very different polarization properties while being spectrally very similar. It has been known for some time [12] that the high-energy spectrum of Cygnus X-1 sometimes has an excess of photons above around $400 \mathrm{keV}$, over and above what is expected from the population of thermal particles associated with the accretion disk around the black hole. Laurent et al have shown that this 
excess is highly polarized. This is not expected if it is a high-energy continuation of the emission associated with the accretion disk, but emission processes such as synchrotron radiation or inverse-Compton radiation, which are produced by the populations of highly energetic electrons that are found in jets, can be very highly polarized [13]. Given that the spectral characteristics of the excess are also consistent with originating in a jet, it seems very hard to evade the conclusion that jet-related emission is being seen directly in the gamma ray regime. (Unfortunately, no high-energy instrument has anything like the angular resolution necessary to make an image of the jet, which would settle the question.)

Laurent et al have not attempted to answer all of the questions raised by these observations. For example, they have not carried out any modeling to investigate whether the gamma-ray emission is consistent with the properties of the radio jet seen by [9], or whether, possibly more likely since the high-energy excess is only seen at certain states of the accretion disk [12], the detectable gamma-ray jet is a transient phenomenon perhaps related to the transient emission seen at radio wavelengths [14] or in TeV gamma-rays [15]. The presence of a transient, powerful jet might help to explain the discrepancy between the jet power inferred by [4] and the appearance of the standard steady jet seen in radio emission, but this question will have to be addressed by future studies. The real significance of [5] is that it is one of the first uses of high-energy polarimetry, and the first to give us a significant improvement in our understanding of the physics of an X-ray binary. We can hope for more work of this nature from existing and planned gamma-ray and X-ray polarimeters in the future.

\section{Figure}




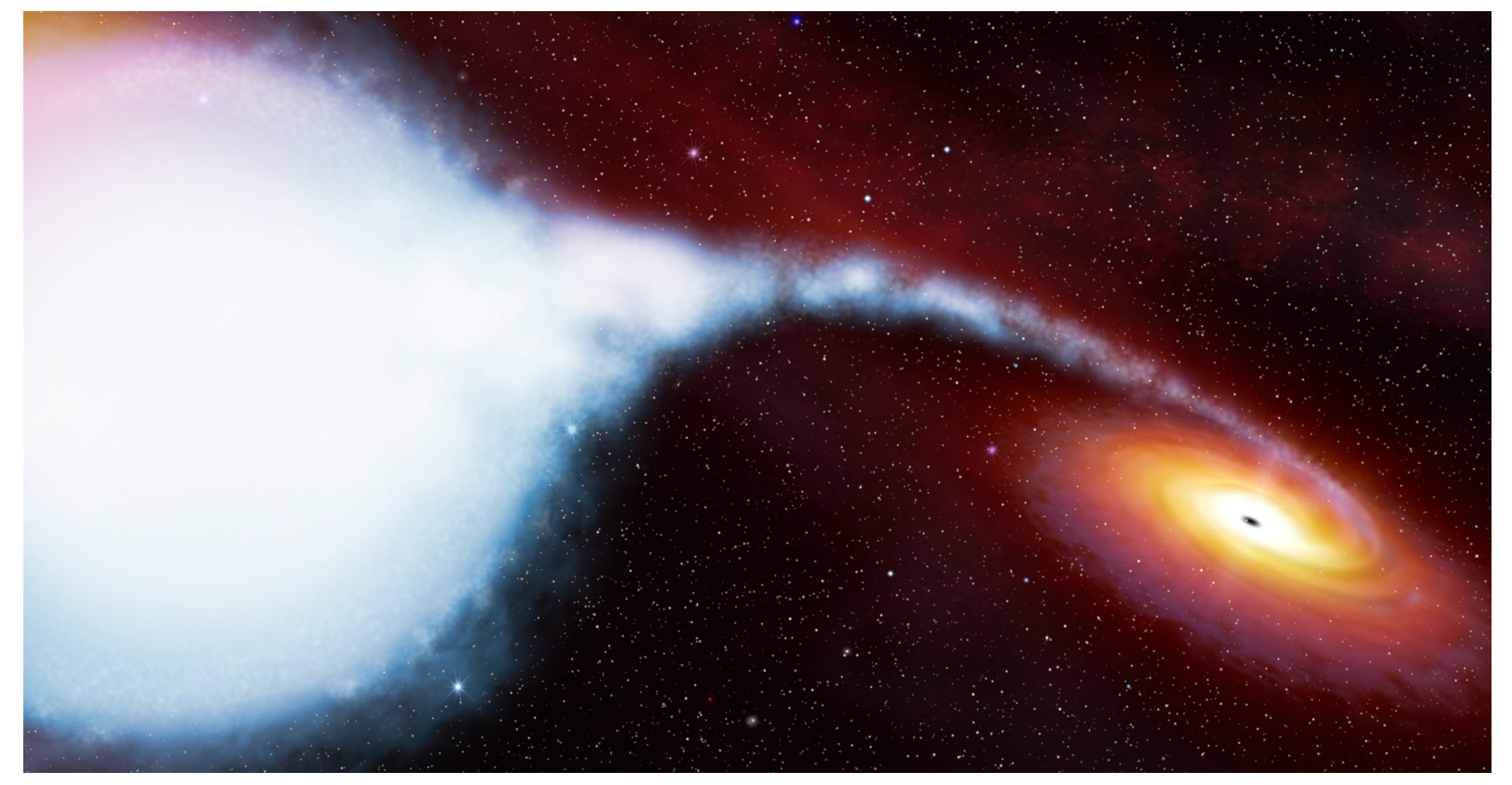

(Taken from http://www.spacetelescope.org/images/cygx1_illustration_orig/)

Caption: An artist's impression of the Cygnus X-1 system. Material from the massive donor star (left) spirals in to form an accretion disk around the black hole (right).

\section{References}

1. R.P. Fender, T.M. Belloni, E. Gallo, Towards a unified model for black hole X-ray binary jets. Mon. Not. R. Astron. Soc, 355, 1105-1118 (2004).

2. R.A. Remillard, J.E. McClintock, X-Ray Properties of Black-Hole Binaries. Annu. Rev. Astron. Astrophys., 44, 49-92 (2006)

3. I.F. Mirabel, L.F. Rodriguez, A superluminal source in the Galaxy. Nature 371, 46-48 (1994)

4. E.R. Gallo et al, A dark jet dominates the power output of the stellar black hole Cygnus X-1. Nature, 436, 819-821 (2005)

5. P. Laurent et al, this issue 
6. S.M. Caballero-Nieves et al., The Ultraviolet Spectrum and Physical Properties of the Mass Donor Star in HD 226868 = Cygnus X-1. Astrophys. J., 701, 1895-1905 (2009).

7. J. Ziółkowski, Evolutionary constraints on the masses of the components of the HDE 226868/Cyg X-1 binary system. Mon. Not. R. Astron. Soc, 358, 851-859 (2005)

8. S. Bowyer, E.T. Byram, T.A. Chubb, H. Friedman, Cosmic X-ray Sources. Science, 147, 394-398.

9. A.M. Stirling et al, A relativistic jet from Cygnus X-1 in the low/hard X-ray state. Mon. Not. R. Astron. Soc, 327, 1273-1278 (2001).

10. S. Heinz, Composition, Collimation, Contamination: The Jet of Cygnus X-1. Astrophys. J., 636, 316-322 (2006)

11. M. Forot, P. Laurent, I. A. Grenier, C. Gouiffés, F. Lebrun, Polarization of the Crab Pulsar and Nebula as Observed by the INTEGRAL/IBIS Telescope. Astrophys. J., 688, L29-32 (2008)

12. M. Cadolle Bel et al, The broad-band spectrum of Cygnus X-1 measured by INTEGRAL. Astron. Astrophys. 446, 591-602 (2006).

13. J. Poutanen, Relativistic jets in blazars: Polarization of radiation. Astrophys. J. Suppl., 92, 607-609 (1994)

14. R.P. Fender et al, A transient relativistic radio jet from Cygnus X-1. Mon. Not. R. Astron. Soc, 396, 603-607 (2006).

15. J. Albert et al, Very High Energy Gamma-Ray Radiation from the Stellar Mass Black Hole Binary Cygnus X-1. Astrophys. J. 665, L51-54 (2007) 